\title{
Pathologies dentaires acquises équines et thérapeutiques
}

\section{Equine dental acquired pathologies and therapeutics}

\section{Résumé}

MOTS-CLEFS :

- Cheval, vétérinaire, technicien dentaire équin, hypsodonte, anisognathie

\section{KEYWORDS:}

- Horse, veterinarian, equine dental technician, hypsodont, anisognathy
Le cheval est un animal herbivore qui possède un estomac simple et fragile. Son appareil masticateur, efficace et performant, permet une bonne assimilation des aliments. Sa dentition, hypsodonte, caractérisée par une croissance dentaire continue associée à une usure des tables occlusales par le mouvement de mastication.

Le développement des sports équestres a relancé la démographie équine et la demande de soins dentaires chez les chevaux. Les soins dentaires courants, réalisés par des vétérinaires ou des techniciens dentaires équins, permettent le maintien d'un équilibre occlusal des arcades indispensable ò une bonne assimilation alimentaire. Les pathologies dentaires plus complexes nécessitent des traitements conservateurs ou chirurgicaux dont les protocoles se rapprochent de la dentisterie humaine et exigent des connaissances plus approfondies que possèdent certains vétérinaires spécialisés.

\author{
Abstract
}

Horse is an herbivorous animal with a simple and fragile stomach. It has also an efficient masticatory system, which enables proper assimilation of food. The hypsodont dentition is characterized by continuing dental growth associated with occlusal wear by the chewing movements.

Equine dental cares are increasingly practiced today through the development of equestrian sports. Routine dental cares, achieved by veterinarians or equine dental technicians, allow maintenance of a balance of occlusal arcades which is essential for a good food assimilation. They also prevent of the occurrence of more complex dental diseases, requiring surgical or conservative treatments. Those protocols are similar to human dentistry and require extensive knowledge possessed only by some specialised veterinarians.

$\operatorname{AOS~n}^{\circ} 273-2015$

Camille FABIER', DDS
Fabienne JORDANA ${ }^{2}, D D S, P h D$
Jacques COLAT-PARROS ${ }^{3}, D D S, P h D$
'Docteur en chirurgie dentaire
Pôle d'Odontologie et de Santé buccale, CHU, Bordeaux, France
UFR d'Odontologie, Université de Bordeaux, Bordeaux, France
'Docteur en chirurgie dentaire, Chirurgien-dentiste, Maître de conférences des Universités, Praticien
hospitalier, Expert près la cour d'appel de Bordeaux
UFR d'Odontologie, Université de Nantes, Nantes, France
Service d'Odontologie, CHU, Nantes, France
3 Docteur en chirurgie dentaire, Maître de Conférences des Universités, Praticien hospitalier, Expert
près la cour d'appel de Pau
Pôle d'Odontologie et de Santé buccale, CHU, Bordeaux, France
UFR d'Odontologie, Université de Bordeaux, Bordeaux, France




\section{$\mathrm{L}$} es premiers écrits sur la dentisterie équine remontent à la civilisation des Hittites (1360 avant J-C). Depuis une trentaine d'années, il existe un regain de demande en soins dentaires pour les chevaux pouvant s'expliquer par l'essor des sports équestres, des courses et de l'élevage. La population d'équidés en France a suivi une augmentation régulière lors de ses deux dernières décennies, pour s'établir aujourd'hui à 900000 équidés selon les données des Haras nationaux.

Le cheval possède une dentition hypsodonte, caractérisée par une croissance dentaire continue associée à une usure des tables occlusales par le mouvement de mastication. Les soins dentaires chez les chevaux, inspirés de la dentisterie humaine, ont été apportés au cours du temps par différentes acteurs : maréchaux-ferrants, vétérinaires et dentistes équins.

\section{ANATOMIE (FIG. 1)}

Os maxillaires et os incisifs constituent la mâchoire supérieure. Sur le bord antérieur de l'os maxillaire, s'implantent les prémolaires et les molaires. L'os incisif présente un processus alvéolaire, incurvé en arc de cercle où s'implantent les trois incisives $[3,4]$. La mandibule est un os pair, asymétrique, formant la mâchoire inférieure. Elle se lie à l'os temporal par l'articulation temporo-mandibulaire (ATM). Le corps de la mandibule porte les dents incisives et éventuellement les canines si elles sont présentes. On retrouve, comme pour l'os maxillaire, le bord inter-alvéolaire où les processus alvéolaires sont absents. En arrière de cette zone, se situent les processus alvéolaires des prémolaires et des molaires $[3,4]$.
On distingue chez le cheval, de chaque côté, cinq sinus (frontal, ethmoïdal, sphénoïdal, maxillaire caudal, maxillaire rostral). Seuls le sinus maxillaire caudal et le sinus maxillaire rostral sont en rapport avec les dents $[5,6]$. Toute affection dentaire parodontale ou endodontique peut induire une pathologie sinusienne d'origine dentaire. L'éruption continue des dents jugales, associée à une résorption du tissu spongieux, va entraîner une augmentation de taille du compartiment latéral de ces sinus maxillaires lors $\mathrm{du}$ développement [7].

La bouche du cheval, cavité initiale du tube digestif, occupée par la langue, se situe entre les deux mâchoires. Ses dimensions sont importantes : hauteur (8 à $9 \mathrm{~cm}$ moyenne), longueur ( $45 \mathrm{~cm}$ en moyenne), largeur $(12$ à $13 \mathrm{~cm})$.

Les lèvres ont un rôle très important dans la préhension des aliments et dans l'aspiration des liquides. La lèvre supérieure a un rôle de tri dans l'ingestion des aliments. Les joues repoussent les aliments sur les faces triturantes des molaires lors de la mastication, notamment par l'action du muscle buccinateur. Le palais constitue la paroi supérieure de la bouche et la sépare des cavités nasales. Le palais est délimité en avant par les incisives supérieures, en arrière par le voile du palais et sur les côtés par les arcades molaires. Le voile du palais, lors de la déglutition, s'oppose au passage des aliments dans les fosses nasales. La région sublinguale (ou canal lingual) constitue le plancher de la bouche.

La langue joue un rôle important dans la sélection des aliments, la déglutition et la préparation digestive du bol alimentaire. Lors de la mastication, elle repousse sans cesse les aliments à triturer sur les prémolaires et molaires.

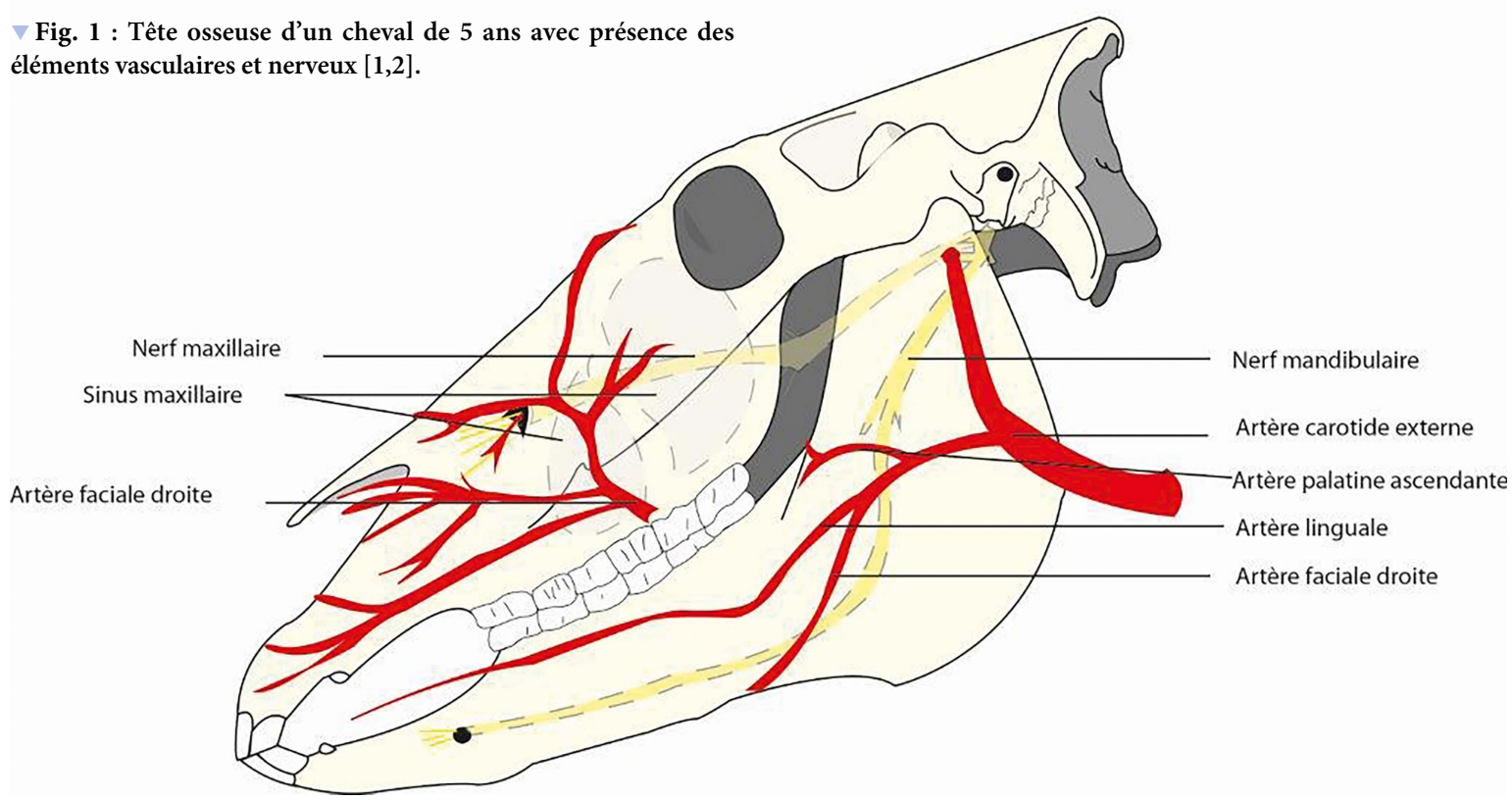


Le cheval sécrète une grande quantité de salive par jour (environ $40 \mathrm{~L} / \mathrm{j}$ ). Il existe de nombreuses petites glandes salivaires au niveau des joues, de la langue, du palais et de la région buccale. Trois glandes salivaires principales sont présentes de chaque côté : la parotide ( $300 \mathrm{~g}$, pouvant produire $50 \mathrm{~mL} / \mathrm{min}$ ), la glande sub-mandibulaire $(60 \mathrm{~g})$, la glande sub-linguale. $[1,8,9]$

Le réseau artériel de la face se fait à partir de l'artère carotide externe, issue d'une division terminale de l'artère carotide commune. L'artère faciale permet l'irrigation de la langue et des lèvres. L'artère maxillaire irrigue la cavité buccale par deux de ses collatérales : l'artère alvéolaire mandibulaire et l'artère alvéolaire maxillaire. Le réseau veineux reprend le même schéma que le réseau artériel avec la veine glosso-faciale qui suit le trajet de l'artère faciale et la veine maxillaire en rapport avec l'artère maxillaire. Le drainage lymphatique se fait vers les nœuds lymphatiques sub-mandibulaires et les nœuds lymphatiques rétro-pharyngiens pour la mâchoire supérieure, et les lymphonœuds sub-mandibulaires pour la mâchoire inférieure.

Les dents et les gencives sont principalement innervées par le nerf trijumeau $(V)$ qui se divise en deux $[1,10$, 11]. Le nerf maxillaire passe par le foramen maxillaire, sous l'œil, puis par le canal infra-orbitaire où des ramifications innerveront les alvéoles des dents de l'arcade supérieure. Il deviendra ensuite le nerf infra-orbitaire une fois sorti du foramen infra-orbitaire. Le nerf mandibulaire se divise en plusieurs branches : le nerf buccal (glandes salivaires et muqueuse buccale), le nerf lingual (langue), et le nerf alvéolaire mandibulaire qui passe par le foramen mandibulaire sur la face mésiale de la mandibule et dont les rameaux innervent les alvéoles des dents de l'arcade inférieure.

\section{Denture et dentition}

\section{Formule dentaire}

Tout comme l'être humain, le cheval possède une denture diphyodonte. À une denture déciduale, succède une denture permanente qui est complète à l'âge de cinq ans.

La denture déciduale se compose de 24 dents (3 incisives et 3 prémolaires par hémi-arcade). Les canines, très rarement présentes, ne sont pas comptabilisées car elles restent le plus souvent sous la gencive. Parfois, la première prémolaire, appelée « dent de loup ", peut être présente sur l'arcade, ainsi le nombre de dents lactéales peut varier de 24 à 26 , voire 28 ; ces premières prémolaires peuvent persister à l'âge adulte [12, 13] La denture définitive d'un cheval adulte se compose de 40 à 42 , voire 44 dents, selon la persistance ou non des premières prémolaires ( 3 incisives, 1 canine, 3 prémolaires et 3 molaires par hémi-arcade). Chez les juments, les canines sont généralement absentes, sauf exceptions auquel cas la jument est dite «bréhaigne ». Le nombre de dents varie alors pour les juments de 36 à 38 , voire 40 dents $[12,13]$. Un espace appelé « barre " est situé entre les incisives et les dents jugales et permet la mise en place du « mors » au contact de la commissure des lèvres, utilisé pour le travail du cheval $[12,13]$. Le système de Triadan est un système de numérotation universel des dents ressemblant à la classification utilisée chez les humains : la numérotation se fait à trois chiffres pour les chevaux et à deux chiffres chez l'homme (Fig. 2) [14].

L'âge d'éruption des dents lactéales ou définitives varie selon les races. Le tableau présente une moyenne d'âge d'éruption (tableau) [15].

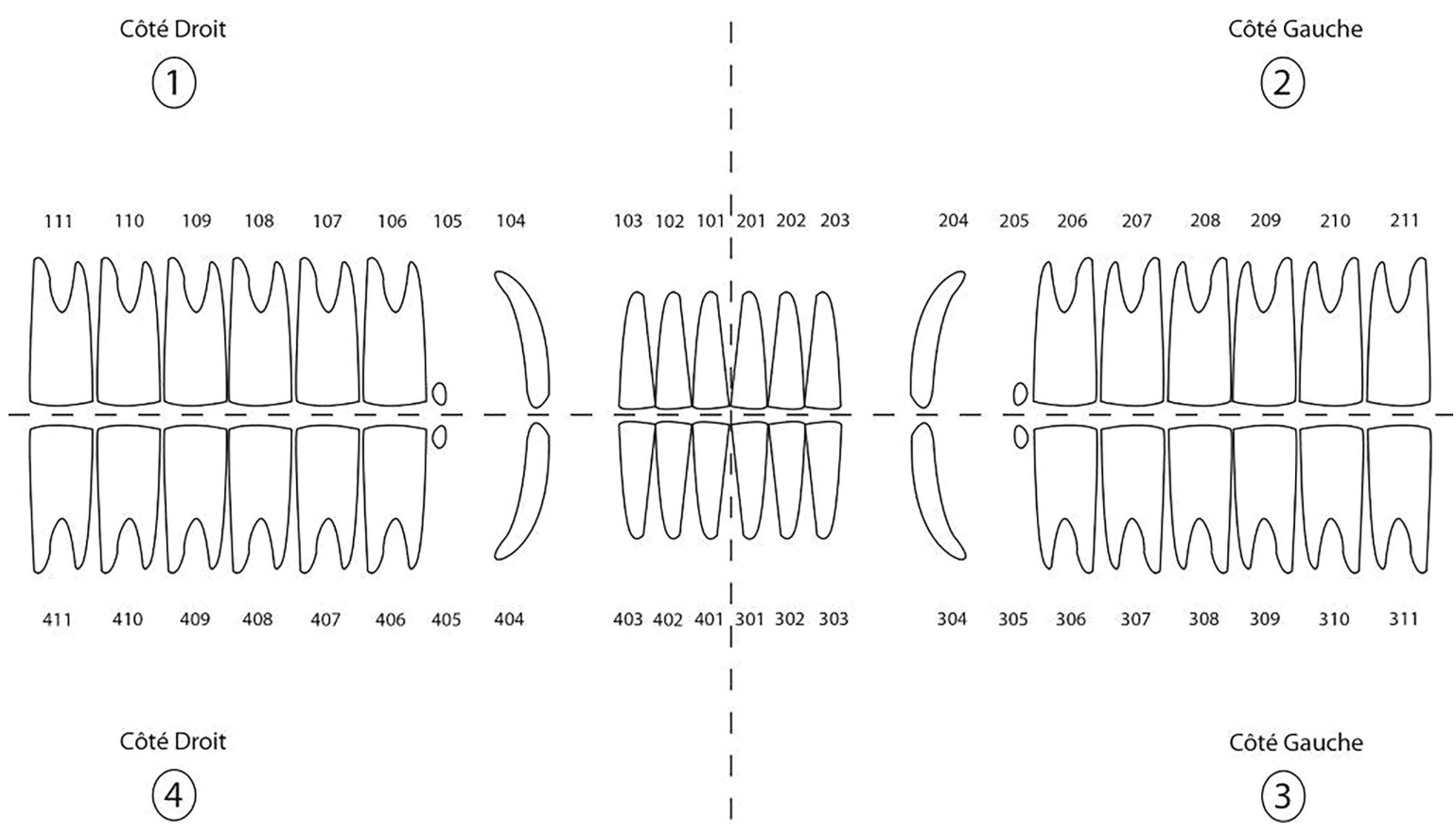

Fig. 2 : 
จ Tableau : Âge moyen d'éruption des dents déciduales et des dents définitives [15].

\begin{tabular}{|l|l|l|}
\hline & Dents déciduales & Dents définitives \\
\hline $1^{\text {re }}$ incisive & à la naissance $-1^{\text {re }}$ semaine & 2 ans $1 / 2$ \\
\hline $2^{\mathrm{e}}$ incisive & 4 semaines & 3 ans $1 / 2$ \\
\hline $3^{\mathrm{e}}$ incisive & 6 mois & 4 ans $1 / 2$ \\
\hline Canine & 6 mois & 4 à 5 ans \\
\hline $1^{\text {re }}$ prémolaire & - & 5 à 6 mois \\
\hline $2^{\mathrm{e}}$ prémolaire & à la naissance $-1^{\text {re }}$ semaine & 2 ans $1 / 2$ \\
\hline $3^{\mathrm{e}}$ prémolaire & $2^{\mathrm{e}}$ semaine & 3 ans \\
\hline $4^{\mathrm{e}}$ prémolaire & $2^{\mathrm{e}}$ semaine & 4 ans \\
\hline $1^{\text {re }}$ molaire & - & 9 à 12 mois \\
\hline $2^{\mathrm{e}}$ molaire & - & 2 ans \\
\hline $3^{\mathrm{e}}$ molaire & - & 3 ans $1 / 2$ à 4 ans \\
\hline
\end{tabular}

\section{Anatomie des dents équines}

À la différence des dents humaines brachyodontes, les dents du cheval sont hypsodontes avec la spécificité d'une couronne très longue, des racines courtes, et une croissance continue de un à deux millimètres par an qui sera compensée par l'usure induite par le processus de mastication.

Sur la face occlusale des incisives et des dents jugales, on peut observer une cavité de couleur grise-jaunâtre remplie de cément et délimitée par une mince couche d'émail appelé cornet dentaire ou infundibulum. Par le phénomène d'usure, ce cornet présent à la naissance disparaitra avec le temps et permettra de donner une estimation de l'âge du cheval [16-19].

Les incisives, au nombre de trois par hémi-arcade, sont dénommées de l'incisive mésiale à la plus distale : "pince », «mitoyenne » et " coin». Les incisives du cheval sont de vrais « chronomètres » de l'âge. Lors de l'éruption, l'édification de la couronne est à peine achevée, une grande partie de la couronne reste enchâssée. L'éruption sera continue tout au long de la vie du cheval. Du fait de l'usure et de cette éruption continue,

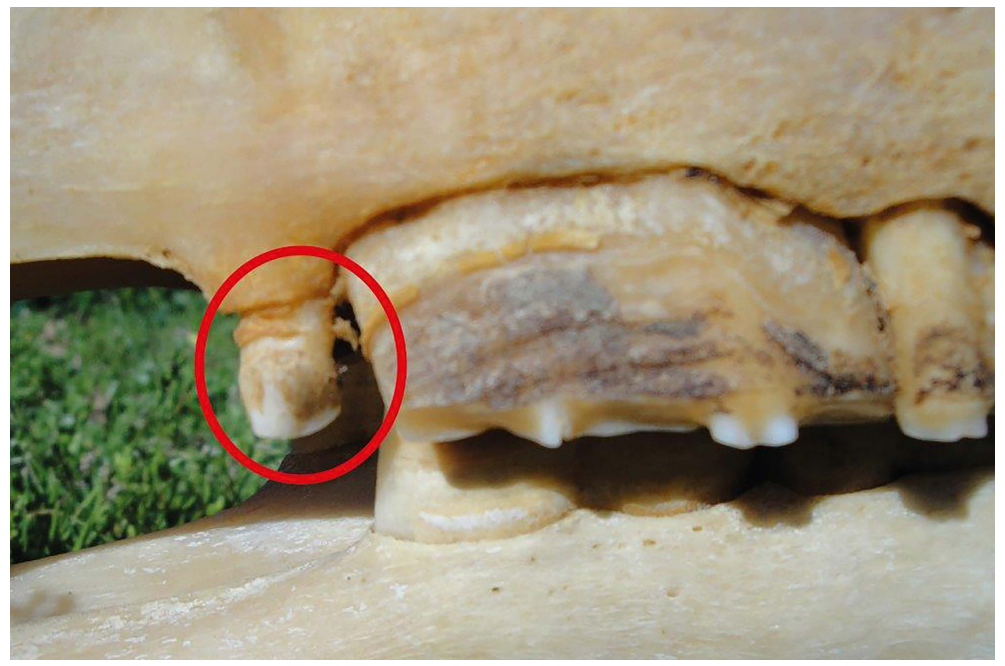

la hauteur de couronne visible reste à peu près stable. La chambre pulpaire se comble de dentine secondaire. L'édification radiculaire complète s'achèvera vers six ans, la racine mesure alors environ 2,5 centimètres $[1,16,17,20]$.

Les canines, aussi appelées " crochets ", sont présentes chez les mâles et très rarement chez les juments ( 2 à $3 \%$ ). Elles peuvent persister à l'intérieur de la mâchoire. De petite taille, ces dents ne sont pas en occlusion et n'ont pas d'utilité dans la mastication. Les canines s'implantent au niveau de la barre à quelques centimètres des «coins» (incisives les plus distales). Les canines inférieures sont situées en avant des canines supérieures. Ces dents, incurvées, présentent une concavité postérieure et une convexité antérieure. Les canines mesurent de 5 à 7 centimètres après éruption complète (vers 6-7 ans) et possèdent une petite couronne dont l'extrémité libre a la forme d'une ogive [1, 21, 22].

Les dents jugales, prémolaires et molaires, présentent une morphologie similaire. Ce sont des dents hypsodontes avec une racine incomplète lors de l'éruption et une usure rapide de la surface occlusale. Le cément est abondant au niveau de la couronne sur les cannelures et dans les sillons de la face linguale provoquant ainsi une usure précoce. La deuxième prémolaire et la dernière molaire ont une forme plutôt triangulaire à sommet mésial pour la deuxième prémolaire et distal pour la dernière molaire à la différence des autres dents jugales de forme carrée ou rectangulaire [1, 16, 20, 23-25].

La première prémolaire supérieure est appelée « dent de loup " (Fig. 3) en référence aux canines des carnivores. Elle se situe en avant des deuxièmes prémolaires et ne possèdent pas de précurseurs en denture temporaire. Tout comme la canine, c'est une dent

$\measuredangle$ Fig. 3 : Dent de loup. 
brachyodonte avec une éruption limitée, une couronne courte, mesurant généralement 10 à 20 millimètres et une racine deux fois plus longue que la couronne. La première prémolaire supérieure est présente chez $90 \%$ des poulains de 6 à 30 mois. Leur incidence naturelle à l'âge adulte varie de 15 à $25 \%$. Ainsi, vers 30 mois, l'éruption de la deuxième prémolaire définitive peut provoquer ou non, selon les individus, la chute de ces "dents de loup ». Certains auteurs appellent «dents de cochon" les premières prémolaires inférieures, généralement plus ovoïdes, qui persistent parfois sous la muqueuse et qui sont beaucoup moins développées que les prémolaires supérieures [26-29].

\section{Occlusion des arcades}

Le cheval présente un anisognathisme : l'arcade supérieure recouvrant l'arcade inférieure. L'arcade maxillaire possède une taille supérieure de 20 à $30 \%$ à l'arcade mandibulaire.

En occlusion physiologique $[23,30]$, proche de la relation centrée, les incisives assurent seules l'occlusion alors que les dents jugales ne sont pas en contact. Lors de mouvements latéraux de la mandibule, les dents jugales entrent en contact du côté travaillant et les incisives sont alors en désocclusion.

\section{Arcades incisives}

Les incisives subissent des modifications tout au long de la vie du cheval. Les arcades incisives ont transversalement une forme parabolique qui évoluera avec le temps en arc de cercle atténué pour devenir rectiligne en fin de vie [1]. L'agencement des arcades de profil va lui aussi se transformer selon trois périodes principales (Fig. 4) [31] :

I Profil en "anse de panier " ou "mors de tricoise" (cheval de moins de 8 ans), les dents définitives du jeune cheval sont en place.

\ Profil « ogival » (cheval entre 8 et 13 ans), les arcades se rétrécissent et prennent la forme d'anse de panier surbaissé.

I Profil « angulaire» (cheval de plus de 13 ans), l'affrontement ogival s'aplatit chez le cheval âgé formant un angle aigu

\section{Correspondance des arcades}

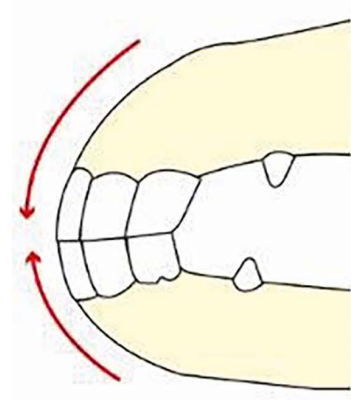

"Mors de tricoise»

Orientation des dents

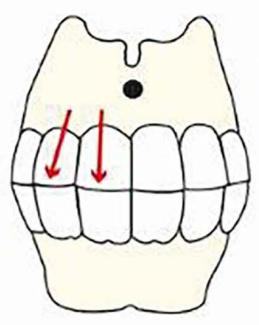

Divergentes
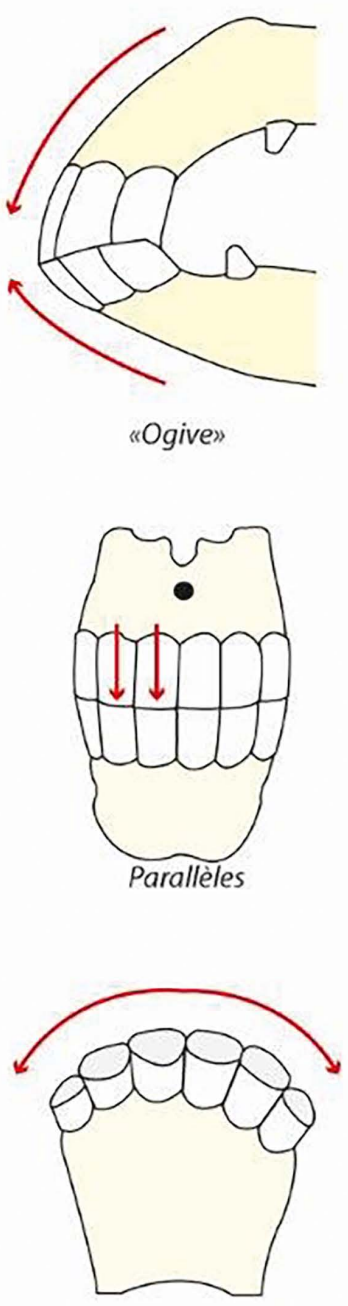

Anse de panier
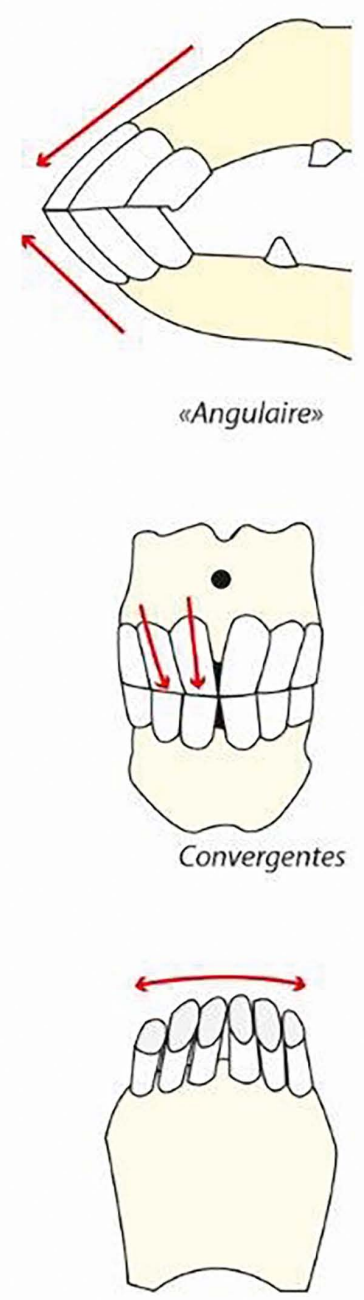

Rectiligne 


\section{Arcades molaires [16, 32, 33]}

Elles ont une longueur de 18 à 20 centimètres. Les arcades supérieures sont légèrement convexes en dehors et convergent à leurs extrémités. Elles débordent les arcades inférieures en dehors. En occlusion, les bords externes des prémolaires et molaires inférieures affrontent les bords internes des prémolaires et des molaires supérieures. La mastication s'effectuera d'un côté à la fois par alternance. Les arcades inférieurs sont plutôt rectilignes et divergent en arrière comme les branches de la mandibule. En vue latérale, les trois dernières dents jugales présentent une concavité supérieure (courbe de Spee). Du fait de l'anisognathisme, les cuspides linguales des prémolaires et molaires mandibulaires sont positionnées juste en-dessous des cuspides palatines des prémolaires et molaires maxillaires. On a donc horizontalement des arcades obliques de haut en bas et de dedans en dehors, avec des surfaces occlusales formant un angle occlusal de 10 à $15^{\circ}$ par rapport à l'axe horizontal des dents. Ainsi, les dents jugales maxillaires forment un angle tranchant côté vestibulaire. Il en est de même pour les dents jugales mandibulaires côté lingual.

\section{PHYSIOLOGIE DIGESTIVE}

\section{Alimentation [34, 35]}

Les chevaux sont des herbivores et granivores. Leur alimentation de base est constituée de fourrage. Selon les besoins, l'état et le travail du cheval, on peut lui apporter un complément alimentaire à base de céréales (orge, avoine, maïs...), de racines et tubercules (carottes, pommes, betterave...). Contrairement aux ruminants, le cheval possède un tractus digestif de type monogastrique, ce qui lui confère un comportement alimentaire spécifique. On préfère donner plusieurs repas par jour au cheval (3 à 4) en raison de la fragilité et la délicatesse de son système digestif $[34,35]$. L'estomac d'un cheval a en effet une faible capacité de 15 à 18 litres. Il est incapable de régurgiter sa nourriture, sauf depuis l'œsophage. La qualité de son alimentation et de sa denture sont des conditions indispensables pour une bonne digestion et pour prévenir les risques pathologiques de type coliques, qui sont la première cause de mortalité équine [34, 35].

\section{Mastication [23, 36]}

Chez la plupart des herbivores, la mastication est longue et énergétique permettant la réduction d'aliments grossiers. La mastication est basée sur la répétition d'un mouvement cyclique résultant de la contraction rythmée des muscles abaisseurs et élévateurs. Elle est la résultante de mouvements de rotation et d'écrasement successivement à droite puis à gauche. Associé à ce mouvement de rotation, l'articulation temporo-mandibulaire fait un mouvement de translation d'arrière en avant respectivement lors de l'ouverture et de la fermeture mandibulaire et lors de la phase de broyage.

La mastication chez le cheval se fait par des mouvements de latéralité de la mandibule, permettant le broyage des aliments par les dents jugales. En relation centrée, les incisives sont les seules dents en contact. Le mouvement de latéralité permet, après désocclusion des incisives, d'initier le contact molaire du côté travaillant.

Le cycle masticatoire comporte trois phases (Fig. 5) [2] : I Phase d'ouverture. La prise d'aliment s'effectue par les incisives après le tri par les lèvres. Une faible amplitude d'ouverture de la mandibule est associée à une diduction à droite ou à gauche qui va initier un contact des molaires et un écrasement de la nourriture.

I Phase de fermeture. La fermeture mandibulaire s'enclenche. Elle est suivie d'un mouvement de diduction ramenant la mandibule médialement, permettant la mise en contact des aliments avec les dents. Les aliments peuvent alors être écrasés.

I Phase de broyage. Elle correspond à la phase de broyage du bol alimentaire par trituration des molaires avec glissement des arcades. La mandibule se déplace par des mouvements diagonaux associant une diduction et une translation d'arrière en avant du côté gauche puis du côté droit.

La mandibule reprend ensuite sa position centrale. L'action rotatoire de la mastication, aidée par la langue et la compression des joues, déplace l'alimentation distalement dans un mouvement de spirale. Le bol alimentaire se collecte ensuite dans l'oro-pharynx puis vers l'œsophage après déglutition.

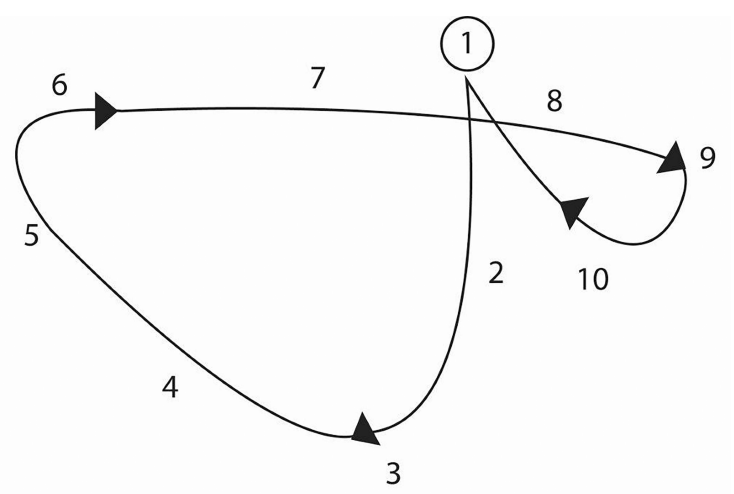

$\triangle$ Fig. 5 : Cycle masticatoire du cheval [2].

- 1 à 4 : phase d'ouverture ;

- 5 à 6 : phase de fermeture ;

-7 à 10 : phase de broyage. 
L'articulation temporo-mandibulaire est une articulation synoviale non congruente qui permet le mouvement de mastication. Sa surface articulaire est plus large dans le sens médio-latéral que chez les humains. Elle est de type diarthrose et permet des mouvements d'élévations et abaissements ainsi que des mouvements de diductions, rétropulsions et propulsions, à un degré moins important que chez l'humain [9, 37-40]

\section{PATHOLOGIES ACQUISES}

\section{Les anomales d'usure [41-48]}

Il est important chez le cheval de surveiller régulièrement les défauts d'occlusion. Ces anomalies peuvent provoquer des difficultés de mastication et d'ingestion des aliments pouvant aboutir à une altération de l'état général à terme.

\section{Régullarisation des tables occlusales postérieures par un nivellement}

Dans une étude réalisée sur 483 chevaux, il apparaît que $86,1 \%$ des chevaux présentaient des défauts d'occlusion. Ces défauts sont majoritairement causés par la présence de pointes d'émail, appelées aussi surdents, qui sont vestibulaires pour l'arcade supérieure (Fig. 6A) et linguales pour l'arcade inférieure [49].

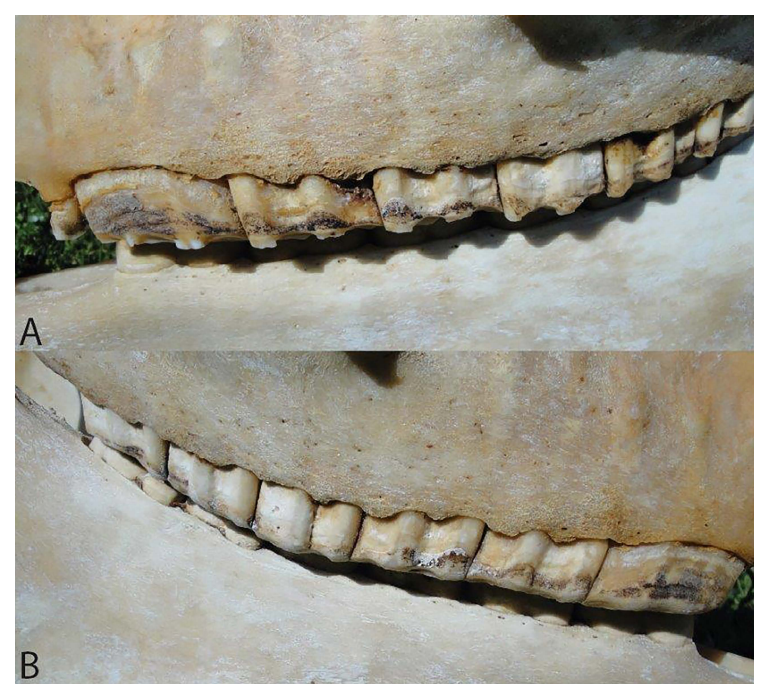

$\triangle$ Fig. 6A : Arcade maxillaire avec de nombreuses pointes d'émail tranchantes en vestibulaire.

Fig. 6B : Arcade dentaire maxillaire lisse sans aspérité après un nivellement.

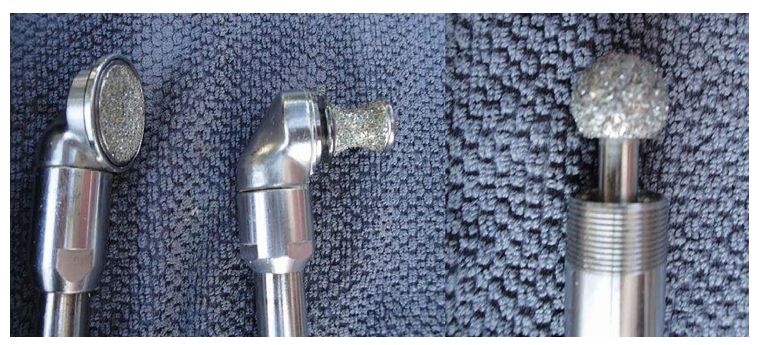

La localisation de ces surdents s'explique par l'anisognathie des arcades. L'existence de ces surdents est due à un défaut d'usure des tables dentaires lors de la mastication. Les défauts d'occlusion plus importants sont liés à la présence de crochets d'émail ou d'occlusion en vagues. D'autres étiologies s'ajoutent alors : anomalie d'éruption, dent absente, dent fracturée, brachygnatisme et prognathisme.

L'anomalie de mâchoire la plus courante chez le cheval est le brachygnatisme avec un décalage plus ou moins important qui ne sera pas forcément pathologique. Dans ce cas, les excroissances d'émail se situeront en mésial des deuxièmes prémolaires supérieures (106-206) et en distal des dernières molaires inférieures (311-411). Des excroissances d'émail sont mises en évidence dans une étude sur $64,3 \%$ des chevaux, avec une forte prévalence pour les dents 106-206 et les dents 311-411 [50].

Les irrégularités des tables dentaires doivent être supprimées par le nivellement dentaire (Fig. 6B et Fig. 7). À l'aide de râpes en carbure de tungstène ou diamantées, manuelles ou motorisées (Fig. 8), le praticien cherche à obtenir une surface occlusale sans aspérités et à redonner un angle occlusal de $15^{\circ}$ environ; les arcades des dents jugales étant obliques de haut en bas et de dedans en dehors dans le plan frontal.

Le cheval sera au préalable sédaté par voie intra-veineuse avec un alpha-2-agoniste et une adjonction

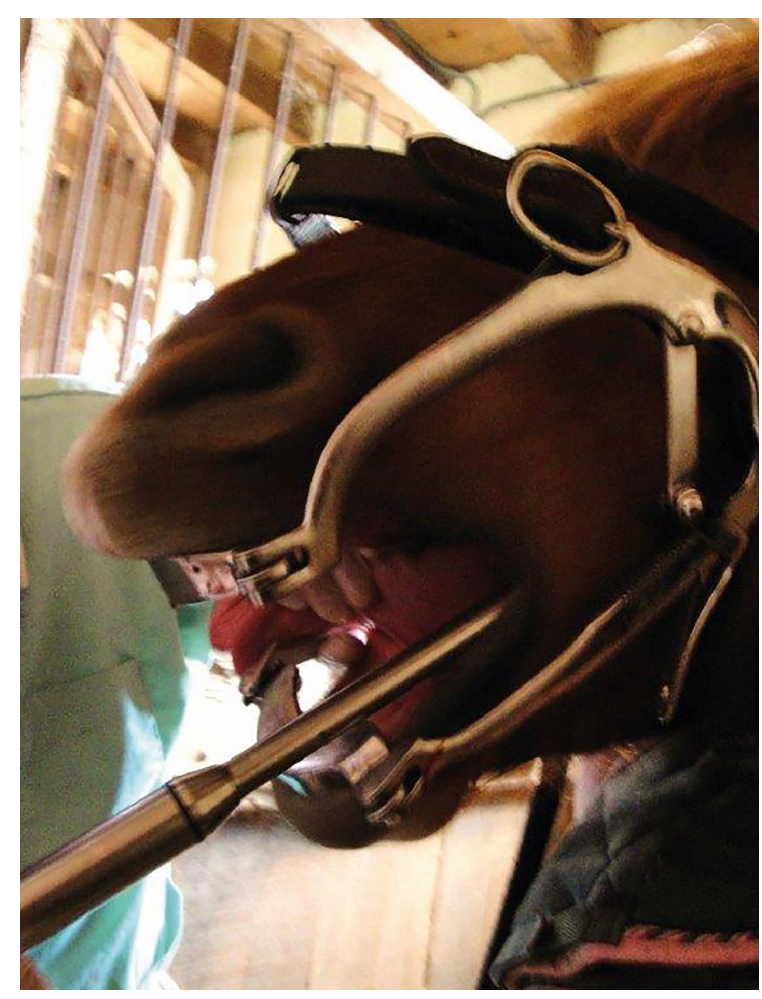

$\triangle$ Fig. $7:$ Technicien dentaire équin pratiquant un nivellement à l'aide d'une râpe motorisée.

$\triangleleft$ Fig. 8 : Exemple de différentes râpes motorisées montées sur moteur. 
d'opioïde pour un maximum de sécurité et de confort à la fois pour le cheval et pour le praticien.

\section{Régularisation des tables incisives}

Les arcades incisives peuvent également présenter, par défaut d'usure ou en cas de fractures ou de dents absentes, des irrégularités de formes variables. L'occlusion peut être en arc de cercle, en diagonale ou en escalier.

Après une sédation, le praticien cherchera à redonner aux arcades un alignement et des contacts corrects à l'aide de disque ou de fraise. La réduction incisive devra être de quelques millimètres seulement afin notamment d'éviter une pathologie pulpaire.

Présence des dents de loups et coiffes déciduales persistantes [51-53]

La dent de loup correspond à la première prémolaire chez le cheval. C'est une dent « rudimentaire » n'ayant aucune utilité dans la mastication de par sa petite taille et sa forme irrégulière variable.

L'action des mains sur les rênes peut amener le mors au contact de ses dents de loup, petites et tranchantes, qui peuvent alors occasionner des blessures au niveau des commissures des lèvres, et des réactions de défense par refus de contact du mors. Il convient alors d'extraire la dent : un élévateur (pouvant être de la forme d'un emporte-pièce cylindrique et tranchant comme l'élévateur de Burgess) permet de mobiliser la dent, puis un davier adapté à manche long permet de finaliser l'extraction (Fig. 9, 10).

La technique nécessite également la sédation du cheval avec adjonction d'une anesthésie locale de type xylocaïne en injection para-apicale avec un rappel palatin (Fig. 11).

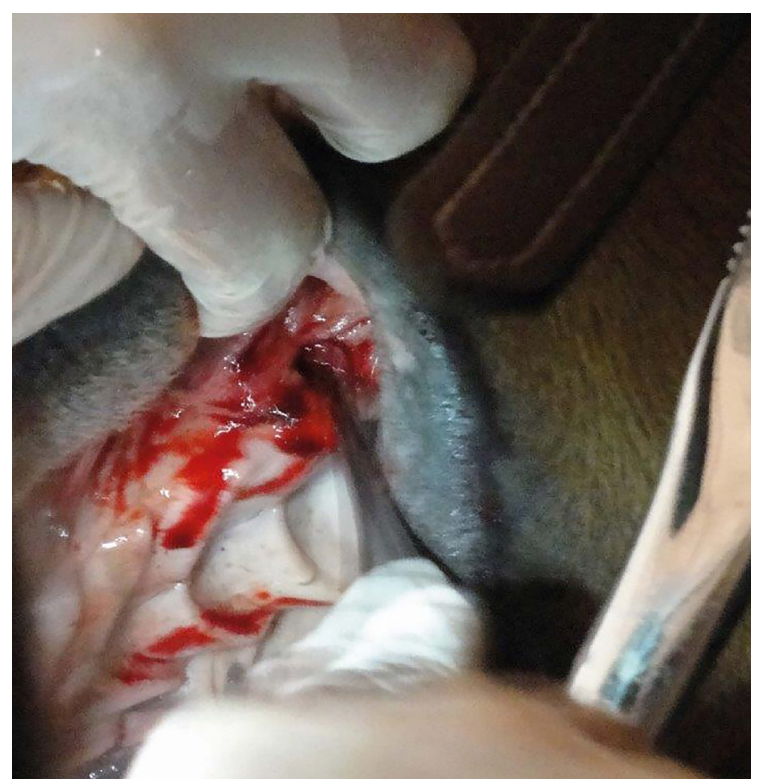

$\triangle$ Fig. 10 : Alvéole dentaire après extraction d'une dent de loup sous-muqueuse.

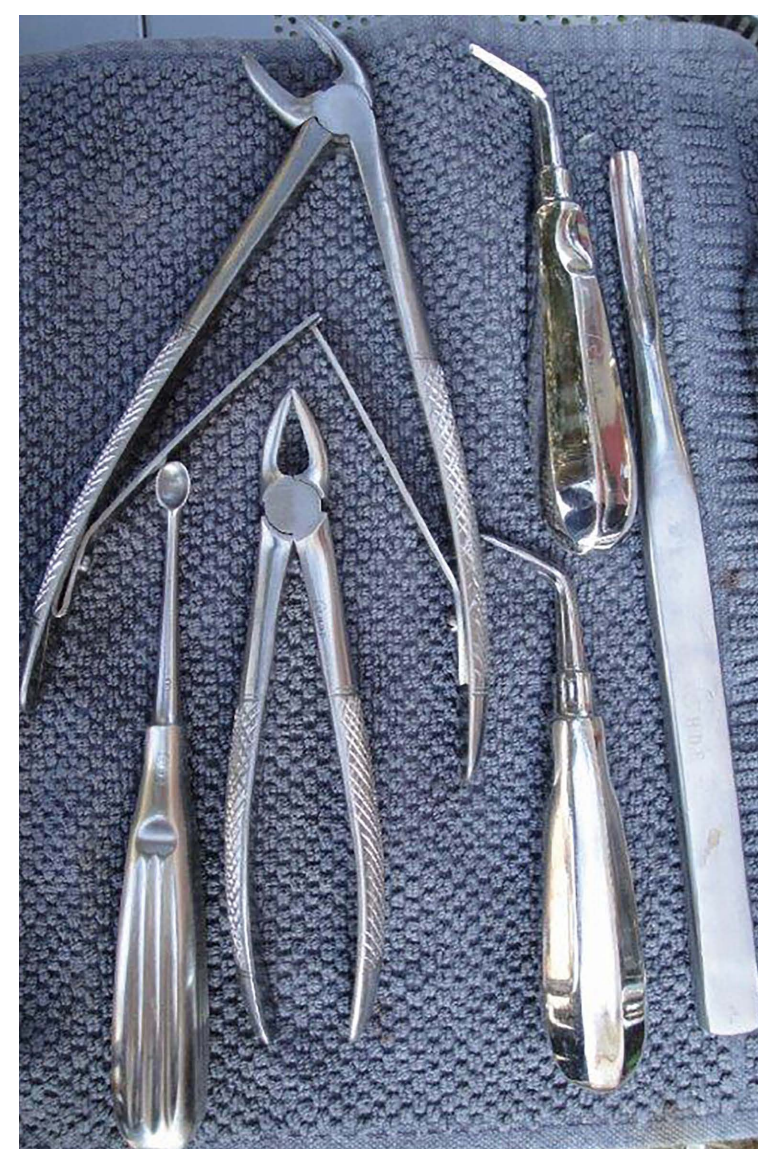

$\triangle$ Fig. 9 : Plateau d'extractions simples.

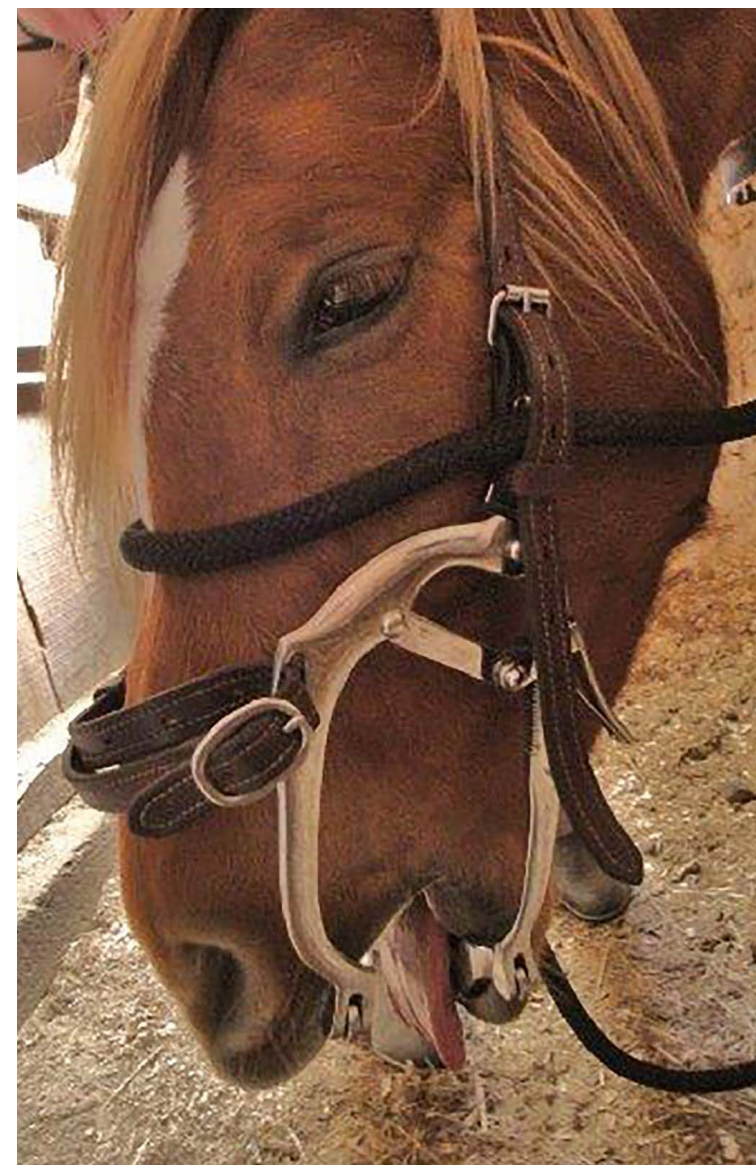

$\triangle$ Fig. 11 : Cheval sédaté avec un pas d'âne en place. 
Entre 2,5 et 4,5 ans, les incisives et les prémolaires définitives font éruption sur les arcades. Les coiffes déciduales peuvent parfois persister et devront être retirées rapidement afin de ne pas perturber la bonne éruption de la denture définitive. Ces dents sont retirées à l'aide d'un davier après sédation du cheval et anesthésie locale.

\section{Pathologies carieuses et endodontiques}

\section{Pathologies carieuses [50, 54-59]}

La carie la plus courante retrouvée chez le cheval se situe au niveau de l'infundibulum des dents jugales par persistance de débris alimentaires à ce niveau. L'hypoplasie du cément est très courante dans cet infundibulum : lors de l'éruption de la dent, l'activité des odontoblastes est stoppée au niveau occlusal et bien souvent la formation de cément ne sera pas achevée. Des zones de vide sont alors présentes dans le cément en profondeur; les risques de caries sont augmentés au fur et à mesure de l'usure de la dent qui découvre ces zones. En l'absence de traitement, une carie peut causer des fractures sagittales de la dent ou encore une atteinte pulpaire malgré la formation de dentine secondaire.

L'incidence serait de 48,3 à $100 \%$ de caries infundibulaires chez des chevaux de douze ans et plus $[50,56$, 59]. Cependant, les études n'étant pas histo-pathologiques, la différence entre hypoplasie du cément et carie ne peut pas être clairement identifiée.

Le traitement de carie suit un protocole identique à la dentisterie humaine : la cavité carieuse sera curetée et nettoyée, puis une obturation sera mise en place de type amalgame, ciment verre ionomère ou composite. Les protocoles et les matériaux sont les mêmes que ceux utilisés en dentisterie humaine.

La croissance continue des dents et l'activité masticatoire puissante chez le cheval peut limiter la viabilité de ces traitements dans le temps avec des récidives possibles. Un suivi régulier sera donc mis en place et le traitement sera à reprendre lors de reprise de carie secondaire à la jonction dent-restauration.

\section{Pathologies endodontiques [60-72]}

L'étiologie principale d'une infection apicale est l'exposition pulpaire induite par une usure trop importante, une fracture dentaire ou une carie profonde. Les signes cliniques sont faibles et difficiles à établir chez le cheval : sensibilité à l'eau froide et difficulté à la mastication. L'examen radiologique est donc indispensable et permet de poser un diagnostic précis.

Le traitement canalaire permet la conservation de la dent avec un maintien de l'occlusion et la continuité d'éruption de la dent qui n'est pas affectée par le traitement. Le traitement endodontique chez le cheval est possible par un accès occlusal classique pour les incisives, les canines et parfois les prémolaires lorsque l'ouverture buccale est suffisante.

Les infections apicales sont peu courantes sur les incisives et sur les canines et vont affecter principalement les dents jugales. Une étude portant sur 400 chevaux donne 3 cas d'infections apicales sur les incisives pour 162 cas pour les dents jugales $[71,72]$.

Pour les dents jugales, une anesthésie générale sera obligatoire pour réaliser la chirurgie endodontique qui présente un taux de réussite de $82 \%$ à 21 mois [73].

Le stade d'infection apicale ne sera pas souvent détecté à temps et pourra évoluer en pathologies loco-régionales : lorsque l'apex de la dent maxillaire communique avec le sinus, un empyème sinusien provoque un jetage nasal unilatéral. Lorsque l'infection apicale évolue en tuméfaction osseuse, celle-ci peut se fistuliser. Dans les deux cas, le praticien pratiquera l'avulsion de la dent pour supprimer tout risque d'infection supplémentaire. Le taux de succès est alors de $81-82 \%[73,74]$.

Chez les jeunes chevaux, les racines sont immatures jusqu'à sept ans environ, le traitement consistera alors à réaliser une apexification avec de l'hydroxyde de calcium dans un premier temps suivie d'un traitement endodontique par la suite, donc avec deux anesthésies générales [74].

\section{Fractures dentaires [75-79]}

On retrouve deux types de fractures chez les chevaux : $\checkmark$ fractures traumatiques : elles sont courantes sur les incisives avec une fracture transversale à la suite de tics, de morsure d'objets ou de congénères, d'action de mors trop forte ou encore de traitements dentaires inadaptés. Sur les dents jugales, on retrouvera ce type de fractures sur les prémolaires principalement. I fractures idiopathiques : l'étiologie de ces fractures n'est pas établie clairement, bien qu'elles soient souvent en rapport avec des caries infundibulaires. Elles touchent les dents jugales longitudinalement, en particulier au milieu des infudibulae.

Ces deux types de fractures sont susceptibles de créer une exposition pulpaire qui pourra évoluer en infection apicale. Leur mise en évidence lors de l'examen clinique est très importante à prendre en compte pour prévenir une infection apicale.

Selon une étude portant sur 50 dents jugales fracturées [78], les fractures traumatiques impliquant les dents jugales mandibulaires sont plus importantes (71\%) que pour les dents jugales maxillaires (29\%). Alors que pour les fractures idiopathiques, l'étude montre $81,5 \%$ de fractures sur les dents jugales maxillaires et $18,5 \%$ sur les dents jugales mandibulaires. 


\section{Pathologies parodontales [80-86]}

L'évolution de la maladie parodontale suit plusieurs étapes :

1) gingivite avec hyperhémie et œdème

12) parodontite avec poche parodontale de moins d'un $\mathrm{cm}$ de profondeur

13) parodontite avec poche parodontale de plus d'un $\mathrm{cm}$ de profondeur

14) parodontite sévère avec des poches parodontales de $3 \mathrm{~cm}$, perte d'os alvéolaire et mobilité dentaire.

On retrouve des problèmes parodontaux environ chez $40 \%$ des chevaux de 3 à 5 ans, $60 \%$ des vieux chevaux à partir de 15 ans en sont atteints [86]. Il existe deux facteurs principaux pouvant initier la maladie parodontale: un défaut d'éruption des dents et une usure anormale des arcades dentaires. Cependant le tassement alimentaire, la plaque, le tartre, l'âge, la présence de bactéries anaérobies, la qualité des défenses immunitaires et l'état général du cheval sont des facteurs influençant également la progression de la maladie. Au niveau de l'espace interdentaire du cheval, une particularité importante concerne le contact interproximal des dents jugales qui est très large. Ainsi la formation de diastèmes accompagnés d'un tassement alimentaire à ce niveau est à surveiller.

Les signes cliniques sont peu visibles au stade débutant. Il convient d'observer une salivation excessive, la présence d'halitose, une mastication difficile ou encore une sensibilité à l'eau froide. Un sondage parodontal permettra de déterminer l'étendue des récessions gingivales, la profondeur des poches parodontales et les mobilités dentaires. Ces éléments seront notés et conservés permettant ainsi d'apprécier l'évolution. La prise de radiographies permet également d'étayer le diagnostic.

Pour les dents jugales, on retrouve fréquemment un tassement alimentaire au niveau inter-proximal en dessous du point de contact, appelé « valve diastema». Concernant les diastèmes primaires, les faces proximales sont parallélisées à l'aide de différentes fraises coniques d'un diamètre de 6 à $8 \mathrm{~mm}$. Dans ce cas, l'ouverture de ces diastèmes permet un auto-nettoyage et une rémission complète 10 mois plus tard pour $72 \%$ des chevaux et une amélioration pour les $28 \%$ restants.

Concernant les diastèmes secondaires, l'étude de Dixon donne, après une intervention en deux temps, un succès pour $48 \%$ des chevaux, $42 \%$ présentant une bonne amélioration, $6 \%$ une faible amélioration et $3 \%$ sans changement [86].

Pour des diastèmes plus larges, ou pour éviter le fraisage chez de jeunes chevaux (risque d'exposition pulpaire), le diastème sera débridé des éléments nécrotiques avec une fraise et comblé avec une résine de type polyméthacrylate de méthyle (PMMA) ou avec une résine composite.

Dans d'autres cas, une contention peut être réalisée en associant une résine de type polyméthacrylate de méthyle (PMMA) avec des fibres de verre.

\section{Autres pathologies [78-90]}

Les tumeurs de la cavité orale sont plutôt rares chez le cheval. Cependant elles sont importantes à prendre en considération lorsqu'elles apparaissent en raison de leur menace pour la survie du cheval.

Les tumeurs d'origine odontogénique sont bénignes et non métaplasiques. Elles sont à évolution lente et chronique. Un examen radiologique et histologique sera nécessaire pour identifier précisément la nature de la tumeur.

Les améloblastomes dérivent de l'épithélium odontogénique. Ce sont des tumeurs sphériques bénignes envahissant localement les tissus environnants. Quel que soit l'âge du cheval, ils apparaissent plutôt à la mandibule, les mâles semblent être plus sujets que les femelles à ce type de tumeur. Cliniquement, la face du cheval peut être déformée avec obstruction nasale possible. Radiologiquement on observe une région kystique centrale radio-claire. Le traitement consiste en exérèse chirurgicale et/ou hémi-mandibulectomie ; une radiothérapie peut y être associée.

Les cémentomes sont d'origine mésodermique, sans composant épithélial et sont localisés à la base de la racine des dents. Ils contiennent des tissus proches $\mathrm{du}$ cément et sont localement invasifs. Ils peuvent être secondaires à une fracture de la dent, à une impaction ou à une atteinte parodontale. Le traitement consiste en l'exérèse chirurgicale.

Les odontomes sont composés de tissus dentaires (émail, dentine, cément et pulpe) avec de l'épithélium et du stroma. La prévalence est plus forte chez le jeune cheval. La région maxillaire des dents jugales est la plus touchée, parfois des kystes sont retrouvés aux sinus. Le traitement de ces malformations bénignes est l'exérèse chirurgicale.

\section{CONCLUSION}

Le système dentaire équin possède des caractéristiques particulières importantes à connaître pour évaluer ses pathologies.

Il existe deux catégories de soins dentaires chez le cheval :

I des soins courants d'entretien avec un effet fonctionnel et prophylactique qui peuvent être réalisés par des techniciens dentaires équins (modification 
du code rural et de la pêche maritime le 22/07/2011, décret d'application du 5/10/ 2011),

I des soins plus complexes donnant lieu à des techniques (protocoles, instruments) exigeant des connaissances plus approfondies. Ces soins seront curatifs et strictement pratiqués par les Vétérinaires équins spécialisés. Ces nombreuses connaissances se sont inspirées de l'odontologie pratiquée chez l'homme. L'application de ces soins restaurateurs, des thérapeutiques endodontiques et des traitements orthodontiques privilégie la conservation des dents.

Des collaborations entre les écoles vétérinaires et les facultés de chirurgie dentaire existent depuis longtemps. Cependant, les Docteurs en chirurgie dentaire ne peuvent en aucun cas intervenir en termes de médecine vétérinaire.

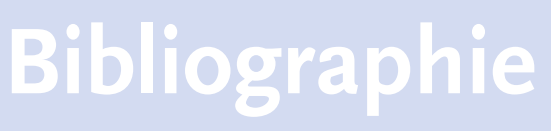

[1] Barone R, Tagand R. Anatomie des équidés domestiques, tome 2 : Splanchnologie et Angéiologie. Lyon : Laboratoire d'Anatomie École Nationale Vétérinaire, 1954.

[2] Easley J et al. Equine dentistry, Third Edition. London : Saunders Elsevier, 2011.

[3] Dacre K. Gross anatomy of the skull. Proc AAEP 2006:1-5

[4] Lardy S. Étude morphométrique de crânes de chevaux de Przewalski en collaboration avec l'association TAKH. Thèse de doctorat vétérinaire, Créteil, 2008.

[5] Cox VS. Peridental Anatomy : Sinuses and mastication muscles. Proc AAEP 2011:1-7.

[6] Schumacher J. Review of anatomy and surgery of the paranasal sinuses of horses. Proc AAEP 2011:165-169.

[7] Crevier-Dx N. Rappels anatomiques : cavités nasales et sinus du cheval. Proc AVEF 2006.

[8] Martin M et al. À Systematic approach to estimating the age of a horse. American Association of Equine Practionners 1999;45:273-275.

[9] P. Pence. Dental anatomy. In : Pence P, ed. Equine dentistry : A Practical Guide. Baltimore : Lippincott Williams\&Wilkins, 2002:1-24.

[10] Flecher BW. How to perform effective equine dental nerve blocks. Proc AAEP 2004:233-236.

[11] Fuss T. Comment réussir une anesthésie d'infiltration en dentisterie ambulatoire? Proc AVEF 2007 :336-339.

[12] Griffin C. Routine dentistry in juvenile performance horses. Compendium Equine : Continuing Education for Veterinarians 2009;4(9):402-415.

[13] Klugh DO. Anatomical characteristics of equine dentition. In : Klugh DO, ed. Principles of equine dentistry. London : Manson publishing, 2010:30-31.
[14] Floyd MR. The modified Triadan system : nomenclature for veterinary system. J Vet Dent 1991;8:18-19.

[15] Martin MT. Guide for Determining the Age of the Horse. 7th ed. Lexington, Ky : AAEP; 2007

[16] Dixon PM. Dental anatomy. In : Baker GJ, Easley J, ed. Equine Dentistry, Second Edition. London : Elsevier Saunders,2005:30-39.

[17] Dixon PM. Dental anatomy. Proc AAEP 2011:8-24.

[18] Dacre IT. Histological and ultrastructural anatomy of equine dentition. Proc AAEP 2006:1-8.

[19] Mitchell SR et al. The structure of peripheral cementum of normal equine cheek teeth. J Vet Dent 2004;21(1):29.

[20] Easley J. Deciduous tooth management. Proc AAEP 2011:141-146.

[21] Caldwell LA. Canine teeth in the equine patient. Proc AAEP 2006:47-55.

[22] Easley J. Equine canine and first premolar (wolf) teeth. Proc AAEP 2004;50:13-18.

[23] Gauchot JY. Dentisterie équine : comprendre pourquoi on nivelle, revues des principales affections dentaires équines nécessitant une réduction. Proc $A V E F$ 2007 :190-204.

[24] Ramzan PHL, Palmer L. Occlusal fissures of the equine cheek tooth. EVJ 2010;42:124-128.

[25] Stubbs RC. Dentistry of equine cheek teeth. Proc AAEP 2004;50:1-6

[26] Banner TA. - Banner TA. Wolf teeth good or bad? Proc NAVC 2005:1-2.

[27] Griffin C. The first premolar teeth. Compendium Equine : Continuing Education for Veterinarians 2009;4:68-76.

[28] Ramzan PHL et al. Chronology and sequence of emergence of permanent premolar teeth in the horse : Study of deciduous premolar'cap' removal in thoroughbred racehorses. EVJ 2009;41:107-111.

[29] Dixon PM, Dacre I. À review of equine dental disorders. Vet J 2005;169:165-187.
[30] Klugh DO. Principles of mastication biomechanics. In : Klugh DO, ed. Principles of equine dentistry. London : Manson publishing, 2010:61-68.

[31] Chatelain E. Dents : diagnose de l'âge des animaux domestiques. Lyon : École Nationale Vétérinaire de Lyon, 1992.

[32] Dixon PM. The Gross, Histological, and Ultrastructural Anatomy of Equine Teeth and Their Relationship to Disease. Proc AAEP $2002 ; 48: 421-437$.

[33] Carmalt JL. Observations of the Cheek Tooth Occlusal Angle in the Horse. J Vet Dent 2004;21:70-5.

[34] Rolland G. L'encyclopédie du cheval. Paris : Aniwa Publishing, 2001.

[35] Sevestre J, Rosier NA. Le cheval. Paris : Larousse, 1983.

[36] Baker GJ. Dental physiology. In : Baker GJ, Easley J, ed. Equine dentistry, $2^{\text {nd }}$ edition. London : Elsevier Saunders, 2005:50-53.

[37] Schumacher J. The equine temporomandibular joint. Proc AAEP 2006:1-5.

[38] Tahier C, Madec S, Lecollinet P, Geffroy O. Un cas d'arthropathie temporo-mandibulaire chez une jument de 17 ans. Proc AVEF 2009.

[39] Klugh DO. Muscles of mastication. In : Klugh DO, ed. Principles of equine dentistry. London : Manson publishing, 2010:57-60.

[40] Weller R et al. The arthroscopic approach and intra-articular anatomy of the equine temporomandibular joint. EVJ 2002;34:421-424.

[41] Allen TE. Incidence and severity of abrasions on the buccal mucosa adjacent to the cheek teeth in 199 horses. Proc AAEP 2004:31-36

[42] Autheville P, Barrairon E. Odontostomatologie vétérinaire. Paris : Maloine, 1985.

[43] Dacre IT. Physiology of mastication. Proc AAEP 2006:15-20.

[44] Delorey MS. À retrospective evaluation of 204 diagonal incisor malocclusion corrections in the horse. J Vet Dent 2007;24:145-149. 
[45] Goubaux A, Barrier G. L'extérieur du cheval. Paris : Asselin et Houzeau, 1890.

[46] Jonhson TJ, Porter C. Dental overgrowths and acquired displacement of cheek teeth/ common disorders of incisor teeth and treatment. Proc AAEP 2006:83-90.

[47] Johnson TJ, Porter C. Dental conditions affecting the mature performance horse (5-15 years). Proc AAEP 2006:17-25.

[48] Tolpeznikovs J. Les abrasions de la muqueuse orale sont-elles toujours associées à des points d'émail tranchants ? Proc AVEF 2006.

[49] Peters JWE et al. Survey of common dental abnormalities in $\mathbf{4 8 3}$ horses in the Netherlands. Proc AAEP 2006:196-202.

[50] Simhofer $\mathrm{H}$ et al. The use of oral endoscopy for detection of cheek teeth abnormalities in 300 horses. Vet $J$ 2008; 178: 396-404.

[51] Chuit P, Gauchot JY. Les soins dentaires courants chez le cheval. Prat Vét Équine 2011;40:23-32.

[52] Griffin C. Extraction of the first premolar teeth. Compendium Equine : Continuing Education for Veterinarians 2009;4:254-266.

[53] Scrutchfield WL. Wolf teeth : how to safely and effectively extract and is it necessary. Proc AAEP 2006:50-60.

[54] Dacre IT. Caries of peripheral cementum. Proc AAEP 2006:91-92.

[55] Dixon PM, Gere I. Postmortem survey of peripheral dental caries in 501 Swedish horses. EVJ 2010;42:310-315.

[56] Honma, K, Yamakawa M. Statistical study on the occurrence of dental caries of domestic animals. Jpn J Vet Res 1962;10:31-36.

[57] Johnson TJ, Porter CM. Infundibular caries. Proc AAEP 2006:101-107.

[58] Ramzan PHL, Palmer L. The incidence and distribution of peripheral caries in the cheek teeth of horses and its association with diastemata and gingival recession. Vet $J$ 2011;190:90-3.

[59] Baker GJ. Some aspects of equine dental decay. EVJ 1974;6:127-130.
[60] Bettiol N, Dixon PM. An anatomical study to evaluate the risk of pulpar exposure during mechanical widening of equine cheek teeth diastemata and "bit seating". EVJ 2011; 43: 163-169.

[61] Dacre IT. Examination for pulp exposure at the occlusal surface and classification of dental fractures. Proc AAEP 2006:1-4.

[62] Dacre IT. Infections apicales et fractures dentaires chez les chevaux. Proc AVEF 2006.

[63] Dacre IT et al. Pathological studies of cheek teeth apical infections in the horse : 4 . Vet J 2008;178:341-51.

[64] Dacre IT et al. Pathological studies of cheek teeth apical infections in the horse : 5 . Vet J 2008;178:352-63.

[65] Dacre IT et al. Pathological studies of cheek teeth apical infections in the horse : 3 . Vet J 2008;178:333-40.

[66] Dixon PM. Acquired disorders of equine teeth. Proc AAEP 2011:93-104.

[67] Dixon PM. Apical infections of cheek teeth and their oral extraction. Proc AAEP 2006.

[68] Shaw DJ et al. Pathological studies of cheek teeth apical infections in the horse : 2 . Vet J 2008;178:321-32.

[69] Velazquez JL. Equine dentistry. Proc WEVA 2009:1-11.

[70] Wilson GJ, Walsh LJ. Temperature changes in dental pulp associated with use of power grinding equipment on equine teeth. Aust Vet J 2005;83:75-7.

[71] Dixon PM et al. Equine dental disease Part 1. EVJ 1999;31:369-77.

[72] Dixon PM et al. Equine dental disease Part 4. EVJ 2000;32:182-94.

[73] Simhofer $\mathrm{H}$ et al. Apicoectomy of maxillary and mandibular cheek teeth in eleven horses - technique and results. Proc AAEP 2006;52:1-8

[74] Wiggs RB, Lobprise H. Veterinary Dentistry : Principles and Practice. Philadelphia : Lippincott-Raven, 1997.

[75] Dacre IT et al. Equine idiopatic cheek teeth fractures. Part 1. EVJ 2007;39: 310-318.
[76] Dacre IT et al. Pathological studies of cheek teeth apical infections in the horse. Vet J 2008;178:311-320.

[77] Dixon PM et al. Idiopathic cheek teeth fractures, including practice-based and hospital-based surveys. Proc AAEP 2006:1-8.

[78] Dixon PM et al. Equine dental disease Part 3. EVJ 2000;32:9-18.

[79] Taylor L, Dixon PM. Equine idiopathic cheek teeth fractures : Part 2. EVJ 2007;39: 322-6.

[80] Baeumlin Y, Vlaminck L. Etude rétrospective sur 47 cas de périodontite : quels enseignements pour la pratique ? Proc AVEF Reims 2008.

[81] Dacre IT. Affections du périodonte des équidés. Proc AVEF 2006.

[82] Greene SK, Basile TP. Recognition and treatment of equine periodontal disease. Proc AAEP 2002;48:463-466.

[83] Johnson TJ, Porter CM. Periodontal disease and tooth decay in the horse. Proc AAEP 2004:19-24.

[84] Klugh DO. Equine periodontal disease. Clin Tech Equine Pract 2005;4:135-147.

[85] Dixon PM et al. Equine dental pathology. In : Easley J, Dixon PM, Schumacher J, ed. Equine dentistry, Third Edition. London : Elsevier, 2011: 129-147.

[86] Dixon PM et al. Treatment of equine cheek teeth by mechanical widening of diastemata in 60 horses (2000-2006). EVJ 2008;40:22-28.

[87] Bouvet $S$ et al. Un cas d'odontome composé chez un poney français de selle de 10 mois. Proc AVEF 2009.

[88] Knottenbelt DC. Oral and dental tumors. In : Baker GJ, Easley J, ed. Equine Dentistry Second Edition. London : Elsevier Saunders, 2005:130-133.

[89] Kutzler MA et al. Mandibular ameloblastoma in a mare. EVE 2007;19: 471-475.

[90] Snyder C et al. Surgical treatment of a rostral mandibular complex odontoma in a 3-year-old horse. EVE 2008;20:647-651. 